\title{
Una estela del Segundo Período Intermedio "reaparecida" en la Colección Fortabat (Buenos Aires, Argentina)
}

\author{
( Diego M. Santos \\ Universidad Pedagógica Nacional, Argentina

\begin{abstract}
Marcelo Campagno
Universidad de Buenos Aires / Consejo Nacional de Investigaciones Científicas y Técnicas, Argentina
\end{abstract}

\section{Introducción ${ }^{1}$}

La Colección de Arte Amalia Lacroze de Fortabat exhibe públicamente una estela conocida por los especialistas desde inicios del siglo XIX, pero hasta ahora no publicada por completo.

La procedencia de la estela es desconocida, aunque posee un notorio pedigree. Aparece por primera vez en la colección del astrónomo y matemático John Lee (1783-1866) en su residencia de Hartwell House y es someramente descripta por Joseph Bonomi el Joven (1796-1878) en 1858 (Bonomi, 1858: 60, $\mathrm{n}^{\circ}$ 450). ${ }^{2}$ Posteriormente pasa a la bien conocida colección de Lord Amherst of Hackney (1835-1909), que será subastada casi veinte años luego de la muerte del coleccionista, en 1921, en Sotheby's de Londres (Sotheby, Wilkinson \& Hodge, 1921: $\left.n^{\circ} 223\right)$. Es en posesión de este coleccionista que será copiada por J. Lieblein (1891: 742, nº 1905). Luego ingresará en la colección del Metropolitan Museum de New York (Inv. 21.2.68) y en esa colección pública será estudiada por William C. Hayes (1978: 346). ${ }^{3}$ En 1956, el Metropolitan Museum la vende a

1 Agradecemos a la Colección Amalita - Colección de Arte Amalia Lacroze de Fortabatla autorización para la publicación de esta estela, así como de la foto amablemente suministrada.

2 Bonomi (1858: 60) la describe del siguiente modo: "Tablet, commencing with the two eyes and the ring. Then follow five lines of hieroglyphics placed horizontally, and then three figures erect in a walking position, two of whom are men. Not a single hieroglyphic wanting". En el margen: "Fine sandstone. 10 3/4 inches high, 7 3/4 inches wide". 
un coleccionista anónimo que a su vez la subastará en Sotheby's de Nueva York en 2006, donde será adquirida por Amalia Lacroze de Fortabat (1921-2012), y destinada a la exhibición pública en 2008. La pieza se encuentra registrada en Topographical Bibliography bajo el número 803-030-490.

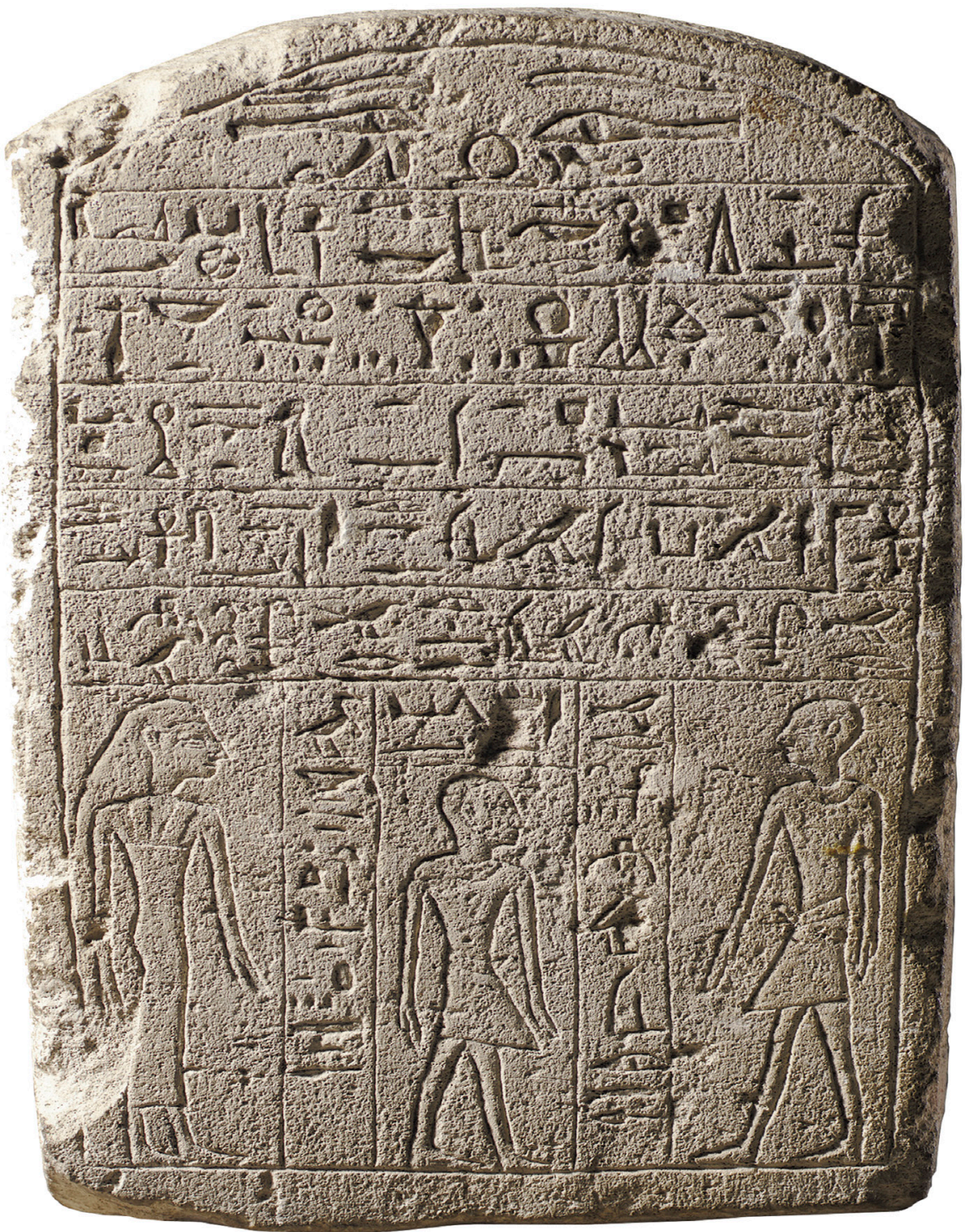

Fig. 1. Estela de Amres.

\section{Descripción}

Se trata de una estela de piedra caliza cuyas medidas: $28,6 \times 20,3 \times 8,9 \mathrm{~cm}$. El borde superior es redondeado. En la parte superior, dos ojos- $w \underline{d} 3 . t$ contrapuestos a ambos lados de un signo- ̌̌n. Presenta cinco líneas de texto en escritura jeroglífica relativamente clara (véase abajo). Debajo hay tres figuras humanas con sus nombres, de izquierda a derecha: una mujer adulta, mirando a der., probablemente un niño de cierta edad (véase abajo), mirando a der., 
y un hombre adulto mirando a izq. Todo se encuentra inciso en la superficie de la piedra. La mujer lleva un vestido y peluca. El presunto niño y el hombre adulto llevan collar-wsh y faldellín. No conserva, de modo evidente, restos de pigmentos, si alguna vez los tuvo.

\section{Inscripción}

La estela presenta una inscripción en cinco líneas (1-5) y tres breves inscripciones (A-B-C) detallando los nombres de los personajes representados.

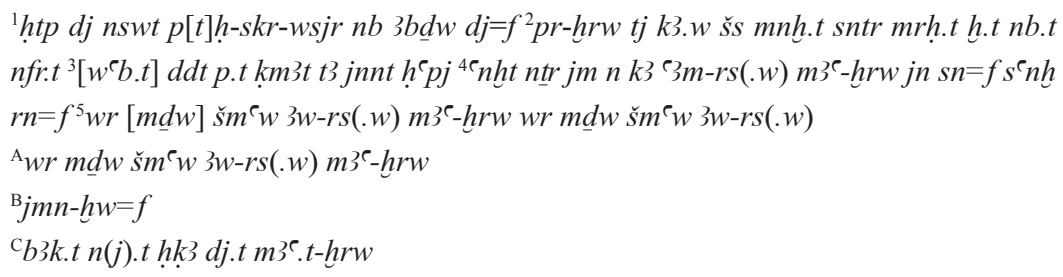

1-5: "Ofrenda que el rey da, y Ptah-Sokar-Osiris señor de Abidos. ${ }^{4}$ Que dé una invocación de ofrendas de pan, bueyes, alabastro, tela, incienso, ungüento y toda cosa buena y pura que da el cielo, que crea la tierra, que trae el Nilo y de la que el dios vive, al $k 3$ (de) $3 m-r s(. w)$ justificado, por su hermano, quien hace vivir su nombre, el Grande de los diez del Alto Egipto, 3w-rs(.w) justificado, el Grande de los diez del Alto Egipto, 3w-rs(.w)".

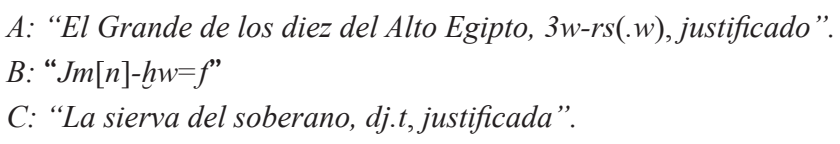

\section{Notas editoriales}

Los faltantes marcados con [] son solo parciales, permitiendo en todos los casos una reconstrucción segura. La lengua de la inscripción es egipcio medio con una tendencia arcaizante no inesperada para la época (véase por ejemplo $n(j) . t, m 3^{\top} . t$ en $\mathrm{C}$, cuando el morfema fem. $-\mathrm{Vt}>-\mathrm{V}$ a fines del Reino Medio, $\mathrm{v}$. Loprieno, 1995: 38; Allen, 2012: 49, sim. /r/ Allen, 2012: 28). El texto tiene algunos errores y omisiones que no reflejan ninguna particularidad lingüística determinada sino, quizás, deficiencias en la formación de los escribas o artesanos.

Ilin-Tomich (2011:33) la ubicó en el sur del Alto Egipto, en el Segundo Período Intermedio, de acuerdo a las características del signo $h t p$.

1.1: htp dj $n(j)-s w . t$ con el ordenamiento de signos del Tipo I de P. Vernus (1991); Marée (2010: 249). Sobre la imprecisión cronológica de este cambio, Franke (2003: 54-55).

4 En la traducción tenemos presentes las sugerencias de Franke (2003) sobre la continuidad en la interpretación de la fórmula entre el Reino Antiguo y el Reino Medio. 
1.2: Se ha cambiado la fórmula $p r-h r w$ tj hnkk.t, como así también omitido 3pd.w, grabando, probablemente por error, $p r-h r w$ tj $k$ 3.w (variante no registrada, v. Ilin-Tomich, 2011: 24-25).

La palabra h.t "cosa" lleva el determinativo Y1 y la marca de plural Z2. Esta ortografía se ha tomado como indicativa para una datación en el Segundo Período Intermedio. Posiblemente, influencia hierática sobre la ortografía jeroglífica (Marée, 2010: 255).

1.4: El adverbio en ' $n h . t ~ n \underline{t}$ $j m$ es reinterpretado como la forma pronominal de la preposición $m(j m=s n)$ en la segunda mitad de la dinastía XVII (El-Shal, 2015: 458; Vernus, 1991: 149; Ilin-Tomich, 2011: 23 con un criterio más amplio, Segundo Período Intermedio).

Falta $n(j)$ luego de $n k$ 3. Probablemente, un error. Las manos del signo $k 3$ están reducidas a círculos rellenos (para ambos fenómenos, v. Marée, 2010: 255-256, común a varios talleres).

jn $s n=f s^{\text {' }} n h \mathrm{~h} r n=f$ "por su hermano, quien hace vivir su nombre". Nelson-Hurst (2010) notó que, durante el Reino Medio, el actor de la Belebungsformel es el hijo. Esta situación cambia entre fines de este período y el Segundo Período Intermedio, siendo el hermano quien ocupa un lugar preponderante a la vez que se incrementan aquellos personajes sin una relación de parentesco especificada. Para Nelson-Hurst, esto evidencia cambios tanto en el mantenimiento del culto funerario (y se aventura a sugerir, también en el plano de la herencia) como en las relaciones sociales.

1.5: En la secuencia $w r m \underline{d} w \check{s} m^{\complement} w 3 w-r s(. w) m 3^{\complement}-h r w w r m \underline{d} w \check{s} m\ulcorner w 3 w-r s(. w)$ no hay vínculo entre ambos $3 w-r s(. w)$. Podía tratarse del mismo nombre duplicado por error o para llenar la línea. También podría estar ausente una relación de filiación expresada por la forma verbal relativa jr.n o el genitivo $s 3$ o $s 3 n(j)$. Adicionalmente, la ortografía en la segunda aparición de $3 w-r s(. w)$ en esta línea se encuentra incompleta. El último signo podría ser -por lo pequeño del último trazo- el determinativo Gardiner A1 en su versión hierática (Möller I $n^{\circ} 33$ ), atestiguando una aparición del nombre $3 w$, aunque lo consideramos poco probable tanto por sus características gráficas como por carecer de determinativo el resto de los nombres de la estela. La falta de espacio puede explicar tanto la ausencia de filiación como lo incompleto del nombre y la falta del correspondiente $m 3^{\complement}$-hrrw.

C. La escritura del signo Gardiner D37 es similar a la hierática más tardía (Möller II n n 103). El trazo medio también está presente en el Gardiner D37 de la 1.1, donde sí se representó el pan. La ortografía corresponde al nombre dj.t . ${ }^{5}$ 


\section{Títulos}

El hermano (y, quizás, el padre) del difunto lleva el título $w r m \underline{d} w s^{\top} m^{`} w$ "Grande de los diez del Alto Egipto". Este título es de difícil interpretación y ha sido repertoriado y discutido varias veces en la bibliografía (Ward, 1982: 87 con bibliografía; Quirke, 1990a: 80; 2009; El-Shal, 2015: 459 con bibliografía) señalando algún tipo de vínculo con la corte. ${ }^{6}$ Teniendo en cuenta los monumentos del período en cuestión, Ilin-Tomich (2015: 128) lo presenta como uno de los títulos "inespecíficos" asociados en el Segundo Período Intermedio especialmente a fuentes sureñas. En monumentos funerarios ("memorial sources"), la élite de las dinastías XVI y XVII parecería preferirlos a los títulos administrativos funcionales (Ilin-Tomich, 2014: 153-154). Para Ilin-Tomich (2014: 157) constituyen "a specific local system of ranking or quasi-ranking titles".

Algo similar sucede con el título femenino presente en la estela, $b 3 k . t n(j) . t$ $h k 3$. Es característico del Alto Egipto y parece tener alguna relación con el masculino $w r m \underline{d} w s^{\varsigma} m^{`} w$. Illin-Tomich (2018: 20-22) cree que posiblemente sea uno de tres títulos llevados por mujeres de la élite sureña según su edad. ${ }^{7}$ Las apariciones en monumentos funerarios de $b 3 k . t n(j) . t h k 3$ corresponden, en su gran mayoría, a madres o esposas del difunto, de donde deduce que es un título llevado por mujeres de edad elevada. Quizás pueda ser alguno de estos vínculos el que caracterice la relación entre el propietario de la estela de la Colección Fortabat y la mujer allí mencionada.

\section{Notas onomásticas, prosopográficas y teóricas}

El nombre del propietario ha sido leído $r s^{8}$ (rs.w “despierto") y, consecuentemente, se creyó que ' $3 \mathrm{~m}$ (usualmente traducido "asiático") era usado aquí como etnónimo (Mourad, 2013). No obstante, la estructura del nombre permite interpretarlo de otra manera. Coincidimos con Persons and Names of the Middle

6 También se pensó que fuera algún tipo de comisionado (Quirke, 1990a: 80). Shirley (2013: 560) señala: "It is possible that the difficult titles (...) represent another connection to the royal court. These have generally been understood as ranking titles possibly pertaining to the vizir's office, which as we have seen does not seem to have functioned after the early 16th Dynasty. The prevalence of these titles on stelae of the Second Intermediate Period, as seen, for example, at Abydos, suggest that during this period they were either functioning on a local level as part of the governor's court, or as representatives of the royal court".

7 La estela de la Colección Fortabat es citada, según su antiguo número del Metropolitan Museum of Art, en relación con este título en Stefanović (2009a: 308 y 2009b: 57-63); Ilin-Tomich (2018: 35).

8 Ranke (1935: I, 226), tomado de esta estela. Para otras apariciones (tanto en hombres como en mujeres), véase la lista en https://pnm.uni-mainz.de/1/name/1165 (accedido el 15-02-2020). Adicionalmente, Louvre C 145 [803-030-490], Louvre C 253 [TB 803-031093], Musée Guimet 2665 [TB 803-030-372] (x2). 
Kingdom ${ }^{9}$ que probablemente deba ser leído $3 m-r s(. w)$ " $3 m$ está despierto", y optamos por esa interpretación, aunque no podemos estar completamente seguros.

Como señala M. Thirion (1981: 82-83), el tipo X-rs(.w)/(.tj) “X está despierto/ despierta" contiene en el primer miembro del compuesto el nombre "d'un parent ou d'un allié, comme a la même époque les noms NN-snb, NN- 'nhww". A la par de estos encontramos variantes con términos genéricos de parentesco como $s n=j-r s(. w)$ "mi hermano está despierto" (Ranke, 1935: I, 308 no 16, 309 $\left.\mathrm{n}^{\circ} 12,310 \mathrm{n}^{\circ} 17\right), j t j=j-r s(. w)$ "mi padre está despierto" (Ranke, 1935: I, 51), $j t j=s-r s(. w)$ "su (f.) padre está despierto" (Ranke, 1935: I, 51) y el más general $r n=f-r s(. w)$ "su (m.) nombre está despierto", $r n=s-r s(. w)$ "su (f.) nombre está despierto" (ambos en Ranke, 1935: I, 223). Un caso que merece un estudio aparte es $r h . w-r s(. w j)$ "los compañeros están despiertos". ${ }^{10}$

Para el Reino Medio tardío, los nombres de esta estructura son un indicativo de origen tebano (Illin-Tomich, 2015: 121 n. 7). En relación con esto, téngase en cuenta el nombre en $B, j m n-h w=f$, que también se ubica en el área de influencia tebana.

El nombre $` 3 m$ (al igual que su femenino $33 m . t$ ) está atestiguado en varias oportunidades en el Reino Medio y el Segundo Período Intermedio (Franke, 1984: 133-134; Schneider, 2003: 7-81; Mourad, 2013; Smith, 1976: 42-43 y 46). El nombre $p 3-3 m$ es también conocido (Ranke, 1935: I, 102). Cabe señalar aquí que dos reyes ubicados por Ryholt (1997) en la dinastía XIV presentan un elemento ${ }^{~} 3 \mathrm{~m}$ en su nombre, con otra ortografía (en escritura de grupo, *`amu): estos son $y^{\ulcorner} 3 m w$ y $3 m w$.

El nombre 3w-rs(.w), por su parte, es raro. Lieblein (1891: 742, n 1905) y Ranke (1935: I, 1, no 9) lo toman de esta estela. Solo estaría atestiguado nuevamente en una estela perdida del Museo de Arqueología de Odessa (Romanova, 2011), con cierto parecido (a juzgar por el único dibujo conservado) con la descripta en este artículo. La estela pertenece al Grande de los diez del Alto Egipto dd.tw, cuyo hermano es $3 w-r s(. w)$, su hija $s 3-m n t w$ y se menciona otro Grande de los diez del Alto Egipto, snb-r-3w. El Persons and Names of the Middle Kingdom lo

9 https://pnm.uni-mainz.de/1/name/3015 (accedido el 15-02-2020) y https://pnm.unimainz.de/1/inscription/2407 (accedido el 15-02-2020).

10 Atestiguado, al menos, en las estelas Wien ÄS 5084 y Moscow l.1.a.5608 (ortografía indubitable), ver https://pnm.uni-mainz.de/1/name/741 (accedido el 15-02-2020). La palabra (Wb. II, 441) está atestiguada desde el Reino Antiguo (Allen, 2017: 246; Hannig, 2003: 728). La referencia a Horus y Seth y a Isis y Neftis (Wb. II, 441; Hannig, 2003: 728; van der Molen, 2000: 284-285) con el nombre rh.wj "los dos compañeros" y rh.tj "las dos compañeras" no debe hacernos suponer que la palabra pueda tener una connotación particular en el campo del vocabulario del parentesco, ya que la especificación rh.wj snsn.wj "compañeros fraternales" (ref. Horus y Seth) en los Textos de los Ataúdes (VI 234f, van der Molen, 2000: 728) parece señalar lo contrario. 
considera dudoso y lo transcribe [.]-rs . ${ }^{11}$ Marée (2010) ubica la producción de la estela perdida en el taller de Abidos que describe.

Los personajes mencionados en la estela de la Colección Fortabat, $3 m-r s(. w)$ y $3 w-r s(w$.$) (su hermano y, también quizás, su padre), recordarían a un 3 m$ y a un $3 w$ con los que compartirían algún vínculo determinado, posiblemente asociado a la esfera del parentesco. De aceptar esa lectura, el ${ }^{*} w$-rs $(. w)$ de la estela perdida de Odessa podría ser el mismo u otro personaje relacionado. El nombre $3 w$ se encuentra atestiguado (Ranke, 1935: I, 1; y quizás en esta estela, véase más arriba). ${ }^{12}$

Aunque hay varios casos contemporáneos (Mourad, 2013), es interesante señalar el posible recuerdo de un pariente o antepasado de origen presumiblemente levantino en el Sur durante el Segundo Período Intermedio en el ámbito de la élite.

En términos interpretativos más generales, vale la pena realizar dos observaciones. Por una parte, tanto $3 w-r s(. w)$ (se trate de uno o dos personajes diferentes) como dj.t se presentan en el texto de la estela por medio de títulos que los asocian a la esfera estatal. En efecto, el título de $3 w-r s(. w), w r m \underline{d} w \check{s} m^{\complement} w$, Grande de los diez del Alto Egipto, conocido desde la Dinastía III (Helck, 1987: 216, 221; Jones, 2000: 388-389), expresa -con independencia de su sentido específico, variable a lo largo del tiempo- la pertenencia a una élite que se define en función del ejercicio del poder político. En cuanto al título de dj.t, $b 3 k . t n(j) . t$ $h k$, sierva del soberano, la presenta a partir de una condición, conocida entre las mujeres de las élites del Alto Egipto de la época, que apunta a un potencial vínculo directo con el monarca. ${ }^{13}$

Por otra parte, toda la escena funeraria transcurre en un contexto palpablemente parental. $3 w-r s(. w)$, en su calidad de oficiante funerario, es el hermano del difunto $3 m-r s(. w)$, y dj.t es probablemente la madre o la esposa de este último. Entre ambos, el personaje de menor altura, identificado como $\mathrm{Jm}[n]$ $h w=f$, podría ser hijo de alguno de los partícipes de la escena funeraria. ${ }^{14}$

11 https://pnm.uni-mainz.de/1/inscription/3391 (accedido el 15-02-2020).

12 Teniendo en cuenta los nombres del tipo $N$-rs (.w) conocidos, y estando el nombre $3 w$ atestiguado, consideramos esta traducción más probable que la brindada en https:// pnm.uni-mainz.de/1/name/3016 "extended of awakedness (who will be alive a long time) (?)", un compuesto exocéntrico ("nfr- $\left.h r^{\prime \prime}\right)$ similar a $3 w$-jb (t. 3w.t-jb "alegría" Wb. I, 4) pero no atestiguado (aunque sí lo está el sustantivo rsw, Wb. II, 451 "das Wachen, die Wachsamkeit", van der Molen, 2000: 287 "watchfulness").

13 La condición de sierva del soberano no necesariamente implica un vínculo real con un monarca determinado, pero claramente apunta a la esfera estatal como ámbito de legitimación. Para el mismo contexto histórico de esta escena, algo similar sucede, de acuerdo con el análisis de Ilin-Tomich (2018: 22-25), con la condición de s3-nswt, "hijo del rey", que frecuentemente exhiben los hijos de mujeres de la élite identificadas como $\underline{h} k r . t$-nswt, "ornamento del rey", un título directamente asociable al de b3k.t n(j).t hk k.

14 Wells (2014: 275-278), quien usa los términos "young children" y "mature children" para distinguir entre aquellos personajes de menor tamaño en relación a hombres y 
Pero además, los propios nombres de los participantes principales -3m-rs(.w) y $3 w-r s(. w)$ - se elaboran a partir de un criterio que, como veíamos, parece asociar al portador del nombre con un pariente o aliado familiar, que parece manifestarse en él. Tomando en cuenta la importancia que los antiguos egipcios atribuían al nombre como una parte esencial de la propia persona (Quirke, 1990b: 9), tal designación implica un fuerte énfasis en la lógica del parentesco como criterio para la definición de la identidad individual.

En este sentido, la Estela de Amres constituye un perfecto ejemplo, en pequeña escala, no solo de la importancia que lo estatal y lo parental poseen en tanto lógicas de organización social en el Antiguo Egipto (Campagno, 2018) sino del carácter articulado a través del que ambas lógicas frecuentemente generaban su efecto de estructuración social en el valle del Nilo.

mujeres adultos. Los primeros presentan las características estereotípicas propias del niño en el arte egipcio. Los segundos están vestidos e identificados con nombres, siendo usualmente mencionados como s3, "hijo", o s3.t, "hija". 


\section{Q Bibliografía}

» Allen, J. P. (2012). The Ancient Egyptian Language. Cambridge: Cambridge University Press.

»Allen, J. P. (2017). A Grammar of the Ancient Egyptian Pyramid Texts. Volume I: Unis. Winona Lake: Eisenbrauns.

»Bonomi, J. (1858). Catalogue of the Egyptian Antiquities in the Museum of Hartwell House. Londres: W. M. Watts.

»Campagno, M. (2018). Lógicas sociales en el Antiguo Egipto. Diez estudios. Buenos Aires: EUFyL.

"El-Shal, O. (2015). La stèle d'loutjéni et les liturgies processionnelles de la fin du Moyen Empire (CGC 20476, GEM 4439), en: Bulletin de l'Institut Français d'Archéologie Orientale 115: 455-470.

»Franke, D. (1984). Personendaten aus dem Mittleren Reich. Wiesbaden: Harrassowitz.

» Franke, D. (2003). The Middle Kingdom Offering Formulas: A Challenge, en: Journal of Egyptian Archaeology 89: 39-57.

» Hannig, R. (2003). Ägyptisches Wörterbuch I. Altes Reich und erste Zwischenzeit. Maguncia: Philip von Zabern.

" Hayes, W. (1978). The Scepter of Egypt: A Background for the Study of the Egyptian Antiquities in The Metropolitan Museum of Art. Vol. 1, From the Earliest Times to the End of the Middle Kingdom. Nueva York: The Metropolitan Museum of Art.

" Helck, W. (1987). Untersuchungen zur Thinitenzeit (ÄA 45). Wiesbaden: Harrassowitz.

» Ilin-Tomich, A. (2011). Changes in the htp-dj-nsw Formula in the Late Middle Kingdom and the Second Intermediate Period, en: Zeitschrift für Ägyptische Sprache 138: 20-34.

" Ilin-Tomich, A. (2014). The Theban Kingdom of Dynasty 16: Its Rise, Administration and Politics, en: Journal of Egyptian History 7: 143-193.

" Ilin-Tomich, A. (2015). Theban Administration in the Late Middle Kingdom, en: Zeitschrift für Ägyptische Sprache 142/2: 120-153.

" llin-Tomich, A. (2018). Female Titles specific to Southern Upper Egypt in the Late Middle Kingdom and Second Intermediate Period, en: The Bulletin of the Australian Centre for Egyptology 26: 19-36.

" Jones, D. (2000). An Index of Ancient Egyptian Titles, Epithets and Phrases of the Old Kingdom (BAR International Series 866). Oxford: Archaeopress.

» Lieblein, J. (1891). Hieroglyphisches Namen-Wörterbuch. Leipzig: J. C. Hinrichs.

»Loprieno, A. (1995). Ancient Egyptian. A Linguistic Introduction. Cambridge: Cambridge University Press.

"Marée, M. (2010). A Sculpture Workshop at Abydos from the Late Sixteenth or Early Seventeenth Dynasty, en: Marée, M. (ed.), The Second Intermediate Period (Thirteenth-Seventeenth Dynasties): Current Research, Future Prospects. Lovaina: Peeters, 241-281. 
» van der Molen, R. (2000). A Hieroglyphic Dictionary of Egyptian Coffin Texts. Leiden: E. J. Brill.

»Mourad, A.-L. (2013). Asiatics and Abydos. From the Twelfth Dynasty to the Early Second Intermediate Period, en: The Bulletin of the Australian Centre for Egyptology 24: 31-58.

»Nelson-Hurst, M. (2010). “...who causes his name to live”: The Vivification Formula through the Second Intermediate Period, en: Hawass, Z. y Houser Wegner, J. (eds.), Millions of Jubilees: Studies in Honor of David P. Silverman. El Cairo: Supreme Council of Antiquities of Egypt, 13-31.

»Quirke, S. (1990a). The Administration of Egypt in the Late Middle Kingdom. New Malden: SIA Publishing.

"Quirke, S. (1990b). Who Were the Pharaohs. London: British Museum Press.

"Quirke, S. (2009). Four Titles: What is the Difference?, en: Silverman, D. P., Simpson, W. K. y Wegner, J. (eds.), Archaism and Innovation: Studies in the Culture of Middle Kingdom Egypt. New Haven: Yale Egyptological Seminar, 305-315.

»Ranke, H. (1935). Die ägyptischen Personennamen. Vol. I. Glückstadt: J. J. Augustin.

» Romanova, O. (2011). Lost Stela of the Odessa Museum of Archaeology, en: Göttinger Miszellen 228: 77-88.

» Ryholt, K. S. B. (1997). The Political Situation in Egypt during the Second Intermediate Period. Copenhague: The Carsten Niebuhr Institute of Near Eastern Studies.

»Schneider, T. (2003). Ausländer in Ägypten während des Mittleren Reiches und der Hyksoszeit. Bd 2. Wiesbaden: Harrassowitz.

"Shirley, J. J. (2013). Crisis and Restructuring of the State: From the Second Intermediate Period to the Advent of the Ramesses, en: Moreno García, J. C. (ed.), Ancient Egyptian Administration. Leiden: E. J. Brill, 521-606.

» Smith, H. S. (1976). The Fortress of Buhen. The Inscriptions. Londres: Egypt Exploration Society.

"Sotheby, Wilkinson \& Hodge (1921). Catalogue of the Amherst collection of Egyptian \& Oriental antiquities, which will be sold by auction by Sotheby, Wilkinson E Hodge... Londres: J. Davy.

"Stefanović, D. (2009a). Stela Bolton 1920.10.12. The Non-Royal Women of the Middle Kingdom II, en: Studien zur altägyptischen Kultur 38: 297-309.

"Stefanović, D. (2009b). The Non-Royal Regular Feminine Titles of the Middle Kingdom and Second Intermediate Period. Londres: Golden House Publications.

» Thirion, M. (1981). Notes d'onomastique. Contribution à une révision du Ranke PN (Deuxième série), en: Revue d'Égyptologie 33: 79-87.

»Vernus, P. (1991). Sur les graphies de la formule "l'offrande que donne le roi" au Moyen Empire et à la Deuxième Periode Intermediaire, en: Quirke, S. (ed.), Middle Kingdom Studies. New Malden: SIA Publishing, 141-152.

"Ward, W. (1982). Index of Egyptian Administrative and Religious Titles of the Middle Kingdom. Beirut: American University of Beirut.

»Wells, E. R. (2014). Display and Devotion: A Social and Religious Analysis of New Kingdom Votive Stelae from Asyut. PhD Dissertation. University of California, Los Angeles. 